Clinical microbiology

\title{
High prevalence of division II (cfiA positive) isolates among blood stream Bacteroides fragilis in Slovenia as determined by MALDI-TOF MS
}

\author{
Samo Jeverica ${ }^{\mathrm{a},{ }^{*}}$, József Sóki ${ }^{\mathrm{b}}$, Manica Mueller Premru ${ }^{\mathrm{a}}$, Elizabeth Nagy ${ }^{\mathrm{b}}$, Lea Papst ${ }^{\mathrm{c}}$ \\ ${ }^{a}$ Institute of Microbiology and Immunology, Faculty of Medicine, University of Ljubljana, Ljubljana, Slovenia \\ ${ }^{\mathrm{b}}$ Institute of Clinical Microbiology, University of Szeged, Szeged, Hungary \\ ${ }^{c}$ Department of Infectious Diseases, University Medical Centre Ljubljana, Ljubljana, Slovenia
}

\section{A R T I C L E I N F O}

Article history:

Received 13 November 2018

Received in revised form

31 January 2019

Accepted 31 January 2019

Available online xxx

\section{Keywords:}

Bacteroides fragilis

Antimicrobial resistance

cfiA carbapenemase

Blood stream isolates

\begin{abstract}
A B S T R A C T
Bacteroides fragilis can be classified into division I (cfiA negative) and division II (cfiA positive) isolates. Division II isolates have a silent chromosomal carbapenemase gene (cfiA) that can become overexpressed by an insertion of a mobile genetic element and thus develop a phenotypic resistance to carbapenems. Aims of our study were (i) to determine the prevalence of $B$. fragilis division II (cfiA positive) isolates among blood stream and non-blood stream isolates from two major Slovenian tertiary-care hospitals and (ii) to assess its influence on phenotypic resistance to imipenem. Consecutive non-duplicate $B$. fragilis isolates from blood stream and non-blood stream specimens were included in the analysis from 2015 to 2017 period. Data from laboratory information system were matched with mass spectra obtained with Microflex LT instrument and MALDI Biotyper 3.1 software (Bruker Daltonik, Bremen, Germany). All mass spectra were reanalyzed using Bruker taxonomy library. Spectra with a $\log$ (score) $>2.0$ were further analyzed with cfiA library that separates $B$. fragilis division I and II isolates based on a $\log$ (score) value difference of $>0.3$. Minimal inhibitory concentrations (MICs) for imipenem were determined with Etest (bioMérieux, Marcy l'Étoile, France), using supplemented Brucella agar and EUCAST breakpoints $(\mathrm{S} \leq 2 \mathrm{mg} / \mathrm{L}, \mathrm{R}>8 \mathrm{mg} / \mathrm{L}$ ). Altogether 623 consecutive B. fragilis isolates were included in the analysis; 47 (7.5\%) were isolated from blood stream and 576 (92.5\%) from non-blood stream specimens. Among all study isolates, 51 ( $8.2 \%$ ) proved to belong to division II (cfiA positive). The proportions of division II isolates among blood stream and non-blood stream isolates were $14.9 \%$ and $7.6 \%$, respectively $(p=0.081$, $\mathrm{ns})$. In total, $1.3 \%(\mathrm{n}=8)$ were non-susceptible to imipenem (MIC $>2 \mathrm{mg} / \mathrm{L}) ; 4.3 \%(\mathrm{n}=2)$ among blood stream and $1 \%(n=6)$ among non-blood stream isolates. All imipenem resistant isolates belonged to division II. Modal MICs (MIC range) were $0.064 \mathrm{mg} / \mathrm{L}(0.016 \mathrm{mg} / \mathrm{L}-2 \mathrm{mg} / \mathrm{L})$ and $0.125 \mathrm{mg} / \mathrm{L}(0.064 \mathrm{mg} / \mathrm{L}-$ $\geq 32 \mathrm{mg} / \mathrm{L}$ ) for division I and II isolates, respectively.
\end{abstract}

() 2019 Elsevier Ltd. All rights reserved.

\section{Introduction}

Bacteroides fragilis is an important human gut commensal bacterium which is also the single most common anaerobic isolate from clinical specimens and can cause a plethora of different human infections such as skin and soft tissue, intraabdominal infections and sepsis [1-3]. This organism is inherently poorly susceptible to a majority of $\beta$-lactam antibiotics but was until

\footnotetext{
* Corresponding author. Institute for Microbiology and Immunology, Medica Faculty, University of Ljubljana, Zaloška 4, SI-1000, Ljubljana, Slovenia.

E-mail address: samo.jeverica@mf.uni-lj.si (S. Jeverica).
}

recently almost fully susceptible to $\beta$-lactam $/ \beta$-lactamase inhibitor combinations and carbapenems $[4,5]$. It can be classified into division I ( $c f i A$ negative) and division II ( $c f i A$ positive) isolates.

The existence of carbapenem-resistant $B$. fragilis was first detected in the late 1980s, soon after the introduction of carbapenems into clinical practice [6,7]. These strains produced $\mathbf{Z n}^{+}$ dependent metallo- $\beta$-lactamase that was able to hydrolyze a majority of $\beta$-lactams, including cephamycins and carbapenems [6], and was coded by the chromosomally mediated cfiA (also known as ccrA) gene [8,9]. It is mostly regarded as a silent gene with a low level of constitutive expression, but its expression can be upregulated following the insertion of an insertion sequence (IS) element 
with an efficient promotor immediately upstream of the gene. This insertion is a one-step genetic event that can occur following antibiotic therapy with carbapenems and is considerably stable even without further selection pressure with carbapenems [10-12]. However, the IS element insertion can explain only about one half of high-level carbapenem resistance in cfiA positive strains while the other half may have other genetic background such as mutations leading to porin loss, upregulation of drug efflux, reduced affinity of penicillin-binding proteins or by some other less well characterized mechanisms [12,13]. Furthermore, it was shown that cfiA positive isolates belong to a distinct homology group of $B$. fragilis also referred to as division II isolates and that division I $B$. fragilis are cfiA negative [14,15]. These two groups of $B$. fragilis isolates are not formal taxonomic entities so far, however they may represent two distinct genospecies or may even represent two groups of $B$. fragilis isolates not yet separated at the species level [15]. Other chromosomal $\beta$-lactamases have been described in $B$. fragilis. CepA is the most widespread among them and confers resistance to penicillins and cephalosporins (except cephamycins). It is inhibited by several $\beta$-lactam inhibitors including clavulanic acid and tazobactam and is the reason why $\beta$-lactam $/ \beta$-lactamase inhibitor combinations retain their activity against most $B$. fragilis isolates. CepA is mainly associated with division I isolates [14,15]. CfXA is a cephalosporinase that is most active against cephaloridine and can be found in several other Bacteroides species in addition to B. fragilis $[16,17]$.

It is unclear whether there are any clinical consequences following an infection with specific division I or II of B. fragilis. Furthermore, there is a paucity of data regarding the epidemiology and antimicrobial resistance of $B$. fragilis in Slovenia and in general. Aims of our study were (i) to determine the prevalence of $B$. fragilis division II (cfiA positive) isolates among blood stream and nonblood stream isolates from two major Slovenian tertiary-care hospitals and (ii) to assess its influence on phenotypic antimicrobial resistance to imipenem.

\section{Methods}

\subsection{Clinical setting and Bacteroides fragilis isolates}

The study was performed at the Institute of Microbiology and Immunology, Medical Faculty, University of Ljubljana, the largest Slovenian microbiology laboratory that serves bacteriological samples from two major tertiary-care hospitals in the area, (i) the University Medical Centre Ljubljana and (ii) the Institute of Oncology Ljubljana, comprising in total around 2400 beds.

The study period was 18 months long, from $07 / 2015$ to $12 / 2017$. From that period, we retrospectively extracted data for all $B$. fragilis isolates from the Institute's laboratory information system. The initial spreadsheet included 1163 isolates with basic patient demographic data, information on collection date, specimen type and antimicrobial susceptibility results, including the MIC values for 5 antimicrobial agents (penicillin, co-amoxiclav, imipenem, clindamycin and metronidazole). From this spreadsheet, we excluded all duplicate isolates, which were defined as isolates from the same patient and specimen type isolated within 30 days period and isolates with identification $\log ($ score $)<2.0$. We further labeled all isolates from blood as blood stream and isolates from other specimen types, majority of which were from wounds and abdominal region, as non-blood stream. The final spreadsheet included 623 consecutive non-duplicate $B$. fragilis isolates.

\subsection{Identification and antimicrobial susceptibility testing}

All isolates were identified with MALDI-TOF mass spectrometry
(MS), using Microflex LT instrument and MALDI Biotyper 3.1 software (Bruker Daltonik, Bremen, Germany). We recovered all $B$. fragilis spectra from the Institute's archive and reanalyzed them with (i) the regular taxonomy library and, furthermore, spectra that corresponded to $B$. fragilis with a $\log$ (score) $>2.0$ with (ii) the "cfiA library" that separates $B$. fragilis division I and II isolates based on a $\log$ (score) value difference of $>0.3$, following the recommendations of the manufacturer. The "cfiA library", provided to us by the Bruker, has two entities ( $c f i A$ positive and $c f i A$ negative) and, was validated previously [15]. The delineation between the two divisions of $B$. fragilis isolates thus involved manipulation and reanalysis of the initially obtained MALDI spectra.

Minimal inhibitory concentrations (MICs) were determined with Etests (bioMérieux, Marcy l'Étoile, France) using supplemented Brucella agar as previously described [3]. EUCAST breakpoints (version 7.1) for penicillin $(\mathrm{S} \leq 0.25 \mathrm{mg} / \mathrm{L}, \mathrm{R}>0.5 \mathrm{mg} / \mathrm{L}$ ), coamoxiclav $(\mathrm{S} \leq 4 \mathrm{mg} / \mathrm{L}, \quad \mathrm{R}>8 \mathrm{mg} / \mathrm{L}), \quad$ imipenem $\quad(\mathrm{S} \leq 2 \mathrm{mg} / \mathrm{L}$, $\mathrm{R}>8 \mathrm{mg} / \mathrm{L})$, clindamycin $(\mathrm{S} \leq 4 \mathrm{mg} / \mathrm{L}, \mathrm{R}>4 \mathrm{mg} / \mathrm{L})$ and metronidazole $(\mathrm{S} \leq 4 \mathrm{mg} / \mathrm{L}, \mathrm{R}>4 \mathrm{mg} / \mathrm{L}$ ) were used for category interpretation. $B$. fragilis ATCC 25286 was used for quality control of the entire susceptibility setting on a weekly basis.

\subsection{Molecular confirmation of cfiA gene and IS element}

During the study period, we prospectively collected $B$. fragilis isolates with imipenem MIC $\geq 0.25 \mathrm{mg} / \mathrm{L}$ for further molecular characterization and thus for confirmation of MALDI-TOF MS cfiA typing results. Isolates were cryopreserved and transported to the Institute of Clinical Microbiology, University of Szeged, Szeged, Hungary, for cfiA gene and activating IS element detection. These experiments were performed as previously described [15], except for $B$. fragilis ID 7, where the upstream activating IS was detected by inverse PCR. This procedure has three steps: (i) digestion of genomic DNA, (ii) ligation to form circular DNAs and (iii) PCR of the inversely ligated DNA. This inverse PCR was essentially carried out as described previously with the following specificities: Bsu RI restriction digestion of chromosomal DNA, I-cfiA-1 (5'-CCATACCCCATCCTTCGATT) and I-cFiA-2 (5'-ATTCACCGATTCACTGACCG) inverse primers and $94{ }^{\circ} \mathrm{C} 3 \mathrm{~min} ; 94^{\circ} \mathrm{C} 15 \mathrm{~s}, 56{ }^{\circ} \mathrm{C} 30 \mathrm{~s}, 72{ }^{\circ} \mathrm{C} 3 \mathrm{~min}$, $35 \mathrm{x} ; 72^{\circ} \mathrm{C} 7 \mathrm{~min}$ inverse PCR cycles [26].

\subsection{Statistical methods}

Basic descriptive statistics was used to describe the sample parameters. Pearson Chi-square test was used for comparison of cfiA positivity prevalence between blood stream and non-blood stream B. fragilis isolates. SPSS Statistics version 23 (IBM Corporation, Armonk, New York, USA) and Microsoft Excel version 16 (Microsoft Corporation, Redmond, Washington, USA) were used for data manipulation and statistical analysis. Statistical significance was set to $p$-value of $<0.05$.

\section{Results}

Altogether, 623 consecutive non-duplicate $B$. fragilis isolates were included in the final analysis, belonging to 596 patients; mean age 66 years (standard deviation: 19 years, range: 0-99 years) of which $48.7 \%(n=290)$ were females. $7.5 \%$ of the isolates $(n=47)$ were from blood stream specimens (blood cultures) and 92.5\% $(n=576)$ were from non-blood stream specimens: $74.2 \%(n=462)$ from wounds and $18.3 \%(n=114)$ from abdominal specimens.

With regard to division I and II distribution, 91.8\% $(n=572)$ of our B. fragilis isolates were division I (cfiA negative) and $8.2 \%$ $(\mathrm{n}=51)$ were division II (cfiA positive). The proportions of division II isolates among blood stream and non-blood stream isolates were 
Table 1

B. fragilis isolates and phenotype distribution.

\begin{tabular}{|c|c|c|c|}
\hline \multirow[t]{2}{*}{ B. fragilis isolates } & Number & Proportion & Division $\mathrm{II}^{\mathrm{b}}$ \\
\hline & $\mathrm{n}$ & $\%$ & $\mathrm{n}(\%)$ \\
\hline Blood stream & 47 & 7.5 & $7(14.9)^{\mathrm{C}}$ \\
\hline Non-blood stream & 576 & 92.5 & $44(7.6)^{c}$ \\
\hline Abdominal $^{\mathrm{a}}$ & 114 & 18.3 & $5(4.4)$ \\
\hline Wound $^{\mathrm{a}}$ & 462 & 74.2 & $39(8.4)$ \\
\hline Total & 623 & 100 & $51(8.2)$ \\
\hline
\end{tabular}

a Non-blood stream isolates.

b Division II (cfiA positive) based on MALDI-TOF MS typing.

c The difference between blood stream and non-blood stream (abdominal+wound) isolates is statistically non-significant $(p=0.081)$.

14.9\% $(n=7)$ and $7.6 \%(n=44)$, respectively. The difference was substantial; however, it did not reach statistical significance $(p=0.081)$. Table 1 shows the distribution of division II isolates among blood stream and non-blood stream $B$. fragilis isolates.

Antimicrobial susceptibility results are shown in Table 2. Nonsusceptibility rates (i.e. resistant and intermediate category combined) among all isolates for penicillin, co-amoxiclav, imipenem, clindamycin and metronidazole were $99.8 \%, 8.3 \%, 1.3 \%, 16.7 \%$ and $0 \%$, respectively. In the subgroup analysis, there was significant difference in clindamycin non-susceptibility rates between blood stream (29.8\%) and non-blood stream (15.6\%) isolates $(p=0.012)$ and significant difference in non-susceptibility rates among division I and II isolates for co-amoxiclav (6.8\% vs $25.5 \%$; $p<0.001)$ and imipenem ( $0 \%$ vs. $15.7 \%$; $p<0.001$ ), respectively. All imipenem resistant isolates belonged to division II. Modal imipenem MICs and MIC ranges were $0.064 \mathrm{mg} / \mathrm{L}$ and $\leq 0.016 \mathrm{mg} / \mathrm{L}-2 \mathrm{mg} / \mathrm{L}$ for division I isolates and $0.125 \mathrm{mg} / \mathrm{L}$ and $0.064 \mathrm{mg} / \mathrm{L}-\geq 32 \mathrm{mg} / \mathrm{L}$ for division II isolates. Fig. 1 shows relative MIC distribution for both division I and II isolates.

A selection of 30 non-duplicate $B$. fragilis isolates with imipenem MIC $\geq 0.25 \mathrm{mg} / \mathrm{L}$ was available for further molecular characterization and detection of cfiA gene and IS element, and thus confirmation of MALDI-TOF MS cfiA typing results. Among them, 24 isolates had MIC $\leq 2 \mathrm{mg} / \mathrm{L}$ and were thus susceptible, two isolates had $\mathrm{MIC}=4 \mathrm{mg} / \mathrm{L}$ (intermediate) and four had MIC $\geq 32 \mathrm{mg} / \mathrm{L}$ (resistant). Seventeen isolates belonged to division II based on MALDI-TOF MS typing and all were confirmed to also harbor the cfiA gene. The concordance between MALDI-TOF MS typing and molecular detection of cfiA gene was $100 \%$ among the selected isolates. IS elements were detected in 2 of the imipenem resistant division II (cfiA positive) isolates. They were identified as IS942 (after inverse PCR of $B$. fragilis ID 7, since the amplification of the upstream fragment of the cfiA gene gave two non-specific fragments) and IS1187 (B. fragilis ID 232) following sequencing. In other 2 imipenem resistant isolates (ID 75 and ID 154), as well as in all other non-resistant isolates, we could not confirm the presence of IS elements. See Table 3 for details.

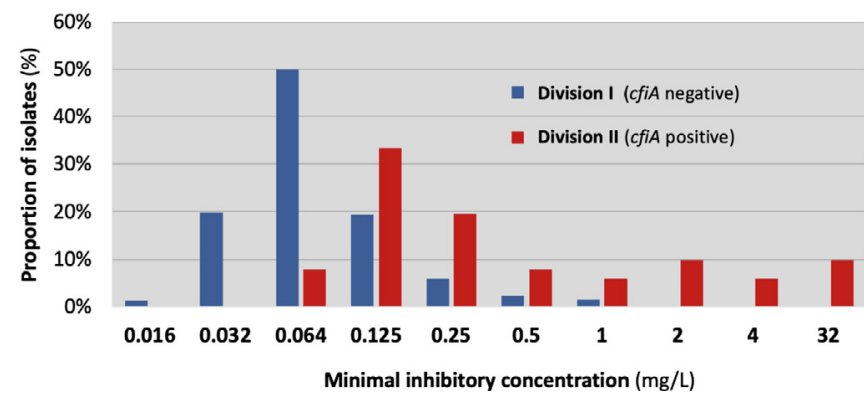

Fig. 1. Relative imipenem MIC distribution among division II (cfiA positive) and division I (cfiA negative) B. fragilis isolates $(n=623)$.

\section{Discussion}

In this study, we have successfully screened a large collection of routine $B$. fragilis isolates $(n=623)$ for phylogenetic division using MALDI-TOF MS. Overall, 8.2\% prevalence of division II isolates was detected in the two largest Slovenian tertiary care hospitals with a marked difference in proportion of division II (cfiA positive) isolates between blood stream and non-blood stream specimens (14.9\% vs. 7.6\%; $p=0.081$ ), respectively. All imipenem resistant isolates belonged to division II. One double dilution higher, but still in the susceptible range, modal imipenem MIC was observed among division II isolates. Imipenem and co-amoxiclav non-susceptibility were linked to division II isolates, while clindamycin nonsusceptibility was more common among blood stream isolates. Perfect concordance between MALDI-TOF MS typing for division I/II discrimination and $c f i A$ gene detection was confirmed in a subset of $B$. fragilis isolates from the study.

Sampling and microbiological technique have a major influence on the isolation of anaerobic bacteria from clinical samples including B. fragilis [18]. For the purpose of anaerobic bacteremia detection, we have used BACTEC Lytic blood culture bottles (Becton Dickinson, Sparks, USA), and for the isolation of anaerobes from other relevant specimen types (i.e. skin and soft tissue and abdominal infections), we have used two anaerobic agar media in combination with thioglycolate broth, namely Schaedler agar (nonselective) and Schaedler Neo. Vanco. agar (selective) (bioMerieux, Marcy-l'Étoile, France, both). A minimum of $48 \mathrm{~h}$ and 5 days anaerobic incubation was used for non-blood culture specimens and blood cultures, respectively. All isolates were identified using MALDI Biotyper 3.1 (Bruker Daltonik, Bremen, Germany) and tested for antimicrobial susceptibility as previously described [3]. Thus, we can be sure that the selection of isolates used in this study is representative of $B$. fragilis infections in our clinical setting.

In order to identify and differentiate between division I and II B. fragilis, we have used a technique described by Nagy and Wybo that is based on MALDI-TOF MS and uses specific main spectra

Table 2

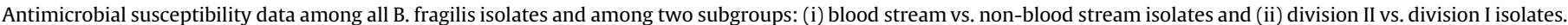

\begin{tabular}{|c|c|c|c|c|c|c|c|c|c|}
\hline \multirow[t]{2}{*}{ Isolates } & \multicolumn{3}{|l|}{ All } & \multirow{3}{*}{$\frac{\frac{\text { Blood stream }}{(n=47)}}{\text { NS }(\%)}$} & \multirow{3}{*}{$\begin{array}{l}\frac{\text { Non-blood stream }}{(\mathrm{n}=576)} \\
\text { NS }(\%)\end{array}$} & \multirow[t]{3}{*}{$p$-value } & \multirow{3}{*}{$\begin{array}{l}\frac{\text { Division II (cfiA pos) }}{(\mathrm{n}=51)} \\
\mathrm{NS}(\%)\end{array}$} & \multirow{3}{*}{$\begin{array}{l}\frac{\text { Division I (cfiA neg) }}{(\mathrm{n}=572)} \\
\mathrm{NS}(\%)\end{array}$} & \multirow[t]{3}{*}{$p$-value } \\
\hline & \multicolumn{3}{|l|}{$(\mathrm{n}=623)$} & & & & & & \\
\hline Antibiotics & $\mathrm{S}(\%)$ & I (\%) & $\mathrm{R}(\%)$ & & & & & & \\
\hline Penicillin & $1(0.2)$ & $0(0)$ & $622(99.8)$ & $47(100)$ & (99.8) & 0.775 & $51(100)$ & $571(99.8)$ & 0.765 \\
\hline Co-amoxiclav & $571(91.7)$ & $12(1.9)$ & $40(6.4)$ & $7(14.9)$ & $45(7.8)$ & 0.091 & $13(25.5)$ & $39(6.8)$ & $<0.001$ \\
\hline Imipenem & 615 (98.7) & $3(0.5)$ & $5(0.8)$ & $2(4.3)$ & $6(1)$ & 0.060 & $8(15.7)$ & $0(0)$ & $<0.001$ \\
\hline Clindamycin & $519(83.3)$ & $0(0)$ & $104(16.7)$ & $14(29.8)$ & $90(15.6)$ & 0.012 & $6(11.8)$ & $98(17.1)$ & 0.325 \\
\hline Metronidazole & $623(100)$ & $0(0)$ & $0(0)$ & $0(0)$ & $0(0)$ & $\mathrm{n} / \mathrm{a}$ & $0(0)$ & $0(0)$ & $\mathrm{n} / \mathrm{a}$ \\
\hline
\end{tabular}

$\mathbf{S}$ susceptible, I intermediate, $\mathbf{R}$ resistant, NS non-susceptible (I+R isolates).

n/a not applicable, pos positive, neg negative. 
Table 3

Concordance between MALDI-TOF MS typing for division I/II discrimination and cfiA gene detection among a selection of $\mathrm{n}=30$ isolates with imipenem MIC $\geq 0.25 \mathrm{mg} / \mathrm{L}$.

\begin{tabular}{|c|c|c|c|c|}
\hline \multirow[t]{2}{*}{ ID } & MIC (imipenem) & MALDI-TOF typing & cfiA gene & IS element \\
\hline & $(\mathrm{mg} / \mathrm{L})$ & (division $\mathrm{I} / \mathrm{II}$ ) & $( \pm)$ & $( \pm)$ \\
\hline 7 & 32 & II & + & $+($ IS942) \\
\hline 75 & 32 & II & + & - \\
\hline 154 & 32 & II & + & - \\
\hline 232 & 32 & II & + & $+($ IS1187) \\
\hline 134 & 4 & II & + & - \\
\hline 220 & 4 & II & + & - \\
\hline 100 & 2 & II & + & - \\
\hline 226 & 2 & II & + & - \\
\hline 230 & 2 & II & + & - \\
\hline 190 & 1 & II & + & - \\
\hline 115 & 0.5 & II & + & - \\
\hline 208 & 0.5 & II & + & - \\
\hline 222 & 0.5 & II & + & - \\
\hline 102 & 0.5 & II & + & - \\
\hline 86 & 0.25 & II & + & - \\
\hline 103 & 0.25 & II & + & - \\
\hline 217 & 0.25 & II & + & - \\
\hline 108 & 1 & $\mathrm{I}$ & - & nt \\
\hline 228 & 1 & I & - & nt \\
\hline 81 & 0.5 & I & - & nt \\
\hline 147 & 0.5 & I & - & nt \\
\hline 166 & 0.5 & I & - & nt \\
\hline 201 & 0.5 & I & - & nt \\
\hline 91 & 0.25 & I & - & nt \\
\hline 93 & 0.25 & I & - & nt \\
\hline 135 & 0.25 & I & - & nt \\
\hline 227 & 0.25 & I & - & nt \\
\hline 233 & 0.25 & I & - & nt \\
\hline 125 & 0.25 & I & - & nt \\
\hline 213 & 0.25 & I & - & nt \\
\hline
\end{tabular}

nt not tested.

a IS insertion sequence element.

library for division discrimination [15,19]. This technique has been proven in several studies to be perfectly concordant with traditional molecular detection of cfiA gene [19-21,24]. It is fast, noncostly, can be applied in real-time following bacterial identification and in different diagnostic settings, including as a direct identification from positive blood cultures bottles [24]. We have used this technique on a prospectively collected mass spectra saved in our database with a batch analysis and thus in a high-throughput manner. However, we also prospectively collected $B$. fragilis isolates with imipenem MIC $\geq 0.125 \mathrm{mg} / \mathrm{L}$ for a molecular confirmation of cfiA gene and IS element in upstream region position and for validation of the entire dataset.

The overall $8.2 \%$ prevalence rate of division II (cfiA positive) isolates was expected and is in concordance with other studies from the same geographic area [20,22,23,25]. However, it is difficult to compare those proportions as it is not clear what the published selections of isolates represent (i.e. consecutive vs. selected isolates) and furthermore it has been obtained from a limited number of isolates. Our collection is among the largest published so far $(\mathrm{n}=623)$ and represents consecutive and non-duplicate isolates [26].

Our main question in this study was whether there was any difference in the prevalence of $c f i A$ positivity between blood stream and non-blood stream isolates. We found a trend that cfiA positive $B$. fragilis are more readily isolated from blood stream samples (14.9\% vs $7.6 \%$ ), yet the difference failed to reach significance $(p=0.081)$. This is in the contrast to the Hungarian study [19], where they surprisingly found no cfiA positive blood culture isolates. Other studies did not report on the proportion differences.

Our phenotypic antimicrobial susceptibility results are in line with our previously published data from 2015 [3]. However, it was very interesting to see the influence of either (i) specimen type or (ii) division I/II type on the cumulative resistance of $B$. fragilis for various tested antibiotics. A major difference in non-susceptibility to imipenem and co-amoxiclav was found among division $\mathrm{I} / \mathrm{II}$ isolates which can be explained, among other reasons, by the fact that division II isolates have cfiA gene which is not always expressed and, that this metallo- $\beta$-lactamase is not inhibited by clavulanic acid. These results should be read with an empirical antibiotic therapy in mind; it may not be safe to treat division II isolates with imipenem and possibly other carbapenems, but it may be even less safe to empirically treat them with co-amoxiclav since approximately one quarter of them are non-susceptible. Interestingly, we have also found almost 2-times higher proportion of clindamycin non-susceptibility among blood stream $B$. fragilis (29.8\% vs. $15.6 \%$ ). This leaves us with extremely narrow options for empiric therapy of $B$. fragilis bacteremia/sepsis where possibly only metronidazole would be acceptable empiric treatment option out of the five antibiotics included in this study.

Several limitations of our study should be outlined. This was a retrospective analysis based on microbiological data only. We do not have any information on either clinical parameters or antimicrobial therapy of the patients. Most importantly, we have no data regarding carbapenem therapy, which is a known selection factor for expression of cfiA gene in division II isolates.

Nevertheless, several implications can be outlined from the results. First, we have confirmed, in the subset of isolates, that division I/II typing is reliable with MALDI-TOF MS and that we can in this way reliably identify $B$. fragilis with silent $c f i A$ gene almost simultaneously with species identification. Second, even though there is still paucity of data that therapy with carbapenems is not indicated for division II $B$. fragilis, it is most probably safe to say that clinicians should be at least careful when treating those infections with carbapenems even if the isolates are phenotypically susceptible and that follow up cultures are warranted when they do so. Third, we would discourage empiric therapy with co-amoxiclav or carbapenems for division II isolates at least in our clinical setting.

In conclusion, a trend towards a higher proportion of division II (cfiA positive) isolates in blood stream specimens as opposed to non-blood stream specimens was detected $(14.9 \%$ vs. $7.6 \%)$ in Slovenia. One double-dilution higher modal imipenem MIC was noticed among division II isolates; however, no clinical implications can be made. Empiric therapy for division II isolates should be locally adjusted and should not include imipenem and possibly also other carbapenems or co-amoxiclav. Further studies focusing on clinical consequences of infections caused by $B$. fragilis division II strains are needed but until then, rapid identification and careful treatment approach, especially for sepsis cases, is warranted.

\section{Ethical approval and informed consent}

Our work did not include any experiments on humans or animals.

\section{Conflicts of interest}

Nothing to declare.

\section{Funding}

This study was supported by the Slovenian Research Agency (Research Programs P3-0083) and institutional department funds.

\section{Acknowledgements}

The authors would like to thank Tinka Lampe from the Institute 
of Microbiology and Immunology, Ljubljana, Slovenia for her meticulous technical assistance during the study and Markus Kostrzewa from Bruker Daltonik GmbH, Bremen, Germany for the cfiA library provision. Two of the authors (SJ, EN) collaborated with the help from the ESCMID Mentorship programme.

\section{References}

[1] H.M. Wexler, Bacteroides: the good, the bad, and the nitty-gritty, Clin Microbiol. Rev. 20 (2007) 593-621, https://doi.org/10.1128/CMR.00008-07.

[2] C.L. Sears, A.L. Geis, F. Housseau, Bacteroides fragilis subverts mucosal biology: from symbiont to colon carcinogenesis, J. Clin. Invest. 124 (2014) 4166-4172, https://doi.org/10.1172/JCI72334.

[3] S. Jeverica, U. Kolenc, M. Mueller-Premru, L. Papst, Evaluation of the routine antimicrobial susceptibility testing results of clinically significant anaerobic bacteria in a Slovenian tertiary-care hospital in 2015, Anaerobe 47 (2017) 64-69, https://doi.org/10.1016/j.anaerobe.2017.04.007.

[4] I. Phillips, A. King, C.E. Nord, B. Hoffstedt, Behalf of a European study group, antibiotic sensitivity of the Bacteroides fragilis group in Europe, Eur. J. Clin Microbiol. Infect. Dis. 11 (1992) 292-304, https://doi.org/10.1007/ BF01962068.

[5] E. Nagy, E. Urban, C.E. Nord, Antimicrobial susceptibility of Bacteroides fragilis group isolates in Europe: 20 years of experience, Clin. Microbiol. Infect. 17 (2011) 371-379, https://doi.org/10.1111/j.1469-0691.2010.03256.x.

[6] G.J. Cuchural, M.H. Malamy, F.P. Tally, Beta-lactamase-mediated imipenem resistance in Bacteroides fragilis, Antimicrob. Agents Chemother. 30 (1986) 645-648, https://doi.org/10.1128/AAC.30.5.645.

[7] K.M. Papp-Wallace, A. Endimiani, M.A. Taracila, R.A. Bonomo, Carbapenems: past, present, and future, Antimicrob. Agents Chemother. 55 (2011) 4943-4960, https://doi.org/10.1128/AAC.00296-11.

[8] B.A. Rasmussen, Y. Gluzman, F.P. Tally, Cloning and sequencing of the class B beta-lactamase gene (ccrA) from Bacteroides fragilis TAL3636, Antimicrob. Agents Chemother. 34 (1990) 1590-1592.

[9] J.S. Thompson, M.H. Malamy, Sequencing the gene for an imipenem-cefoxitinhydrolyzing enzyme (CfiA) from Bacteroides fragilis TAL2480 reveals strong similarity between CfiA and Bacillus cereus beta-lactamase II, J. Bacteriol. 172 (1990) 2584-2593.

[10] I. Podglajen, J. Breuil, E. Collatz, Insertion of a novel DNA sequence, 1S1186, upstream of the silent carbapenemase gene cfiA, promotes expression of carbapenem resistance in clinical isolates of Bacteroides fragilis, Mol. Microbiol. 12 (1994) 105-114.

[11] R. Edwards, P.N. Read, Expression of the carbapenemase gene (cfiA) in Bacteroides fragilis, J. Antimicrob. Chemother. 46 (2000) 1009-1012.

[12] P.-L. Ho, C.-Y. Yau, Y. Wang, K.-H. Chow, Determination of the mutation prevention concentration of imipenem for the two imipenem susceptible Bacteroides fragilis strains Q1F2 ( cfiA -positive) and ATCC 25282 ( cfiA -negative), Int. J. Antimicrob. Agents (2017) 1-6, https://doi.org/10.1016 j.ijantimicag.2017.08.004.

[13] J. Sóki, Extended role for insertion sequence elements in the antibiotic resistance of Bacteroides, World J. Clin. Infect. Dis. 3 (2013) 1-12, https:// doi.org/10.5495/wjcid.v3.i1.1.
[14] R. Ruimy, I. Podglajen, J. Breuil, R. Christen, E. Collatz, A recent fixation of cfiA genes in a monophyletic cluster of Bacteroides fragilis is correlated with the presence of multiple insertion elements, J. Bacteriol. 178 (1996) 1914-1918.

[15] E. Nagy, S. Becker, J. Sóki, E. Urbán, M. Kostrzewa, Differentiation of division I (cfiA-negative) and division II (cfiA-positive) Bacteroides fragilis strains by matrix-assisted laser desorption/ionization time-of-flight mass spectrometry, J. Med. Microbiol. 60 (2011) 1584-1590, https://doi.org/10.1099/jmm.0. 031336-0.

[16] A.C. Parker, C.J. Smith, Genetic and biochemical analysis of a novel Ambler class A beta-lactamase responsible for cefoxitin resistance in Bacteroides species, Antimicrob. Agents Chemother. 37 (1993) 1028-1036.

[17] J. Sóki, M. Hedberg, S. Patrick, B. Bálint, R. Herczeg, I. Nagy, D.W. Hecht E. Nagy, E. Urbán, Emergence and evolution of an international cluster of MDR Bacteroides fragilis isolates, J. Antimicrob. Chemother. 71 (2016) 2441-2448, https://doi.org/10.1093/jac/dkw175.

[18] E. Nagy, L. Boyanova, U.S. Justesen, How to isolate, identify and determine antimicrobial susceptibility of anaerobic bacteria in routine laboratories, Clin. Microbiol. Infect. 24 (2018) 1139-1148, https://doi.org/10.1016/ j.cmi.2018.02.008.

[19] I. Wybo, A. De Bel, O. Soetens, F. Echahidi, K. Vandoorslaer, M. Van Cauwenbergh, et al., Differentiation of cfiA-negative and cfiA-positive Bacteroides fragilis isolates by matrix-assisted laser desorption ionization-time of flight mass spectrometry, J. Clin. Microbiol. 49 (2011), https://doi.org/10.1128/ JCM.02321-10, 1961-1964.

[20] K.P. Sarvari, J. Sóki, M. Iván, C. Miszti, K. Latkóczy, S.Z. Melegh, et al., Detection of enterotoxin and protease genes among Hungarian clinical Bacteroides fragilis isolates, Anaerobe 48 (2017) 98-102, https://doi.org/10.1016/j.anaerobe. 2017.07.005.

[21] S.A. Ferløv-Schwensen, T.V. Sydenham, K.C.M. Hansen, S.V. Hoegh, U.S. Justesen, Prevalence of antimicrobial resistance and the cfiA resistance gene in Danish Bacteroides fragilis group isolates since 1973, Int. J. Antimicrob. Agents 50 (2017) 552-556, https://doi.org/10.1016/j.ijantimicag.2017.05.007.

[22] A.A. Kangaba, F.Y. Saglam, H.B. Tokman, M. Torun, M.M. Torun, The prevalence of enterotoxin and antibiotic resistance genes in clinical and intestinal Bacteroides fragilis group isolates in Turkey, Anaerobe 35 (2015) 72-76, https:// doi.org/10.1016/j.anaerobe.2015.07.008.

[23] E. Székely, Z. Eitel, S. Molnár, IÉ. Szász, D. Bilca, J. Sóki, Analysis of Romanian Bacteroides isolates for antibiotic resistance levels and the corresponding antibiotic resistance genes, Anaerobe 31 (2015) 11-14, https://doi.org/ 10.1016/j.anaerobe.2014.09.001.

[24] Å. Johansson, E. Nagy, J. Sóki, Instant screening and verification of carbapenemase activity in Bacteroides fragilis in positive blood culture, using matrixassisted laser desorption ionization-time of flight mass spectrometry, J. Med. Microbiol. 63 (2014) 1105-1110, https://doi.org/10.1099/jmm.0. 075465-0.

[25] V.S. Fenyvesi, E. Urbán, N. Bartha, M. Abrók, M. Kostrzewa, E. Nagy, et al., Use of MALDI-TOF/MS for routine detection of cfiA gene-positive Bacteroides fragilis strains, Int. J. Antimicrob. Agents 44 (2014) 474-475, https://doi.org/ 10.1016/j.ijantimicag.2014.07.010.

[26] M. Cordovana, M. Kostrzewa, J. Sóki, E. Witt, S. Ambretti, A.B. Pranada, Bacteroides Fragilis: A Whole MALDI-Based Workflow from Identification to Confirmation of Carbapenemase Production for Routine Laboratories, Anaerobe (2018), https://doi.org/10.1016/j.anaerobe.2018.04.004. 